Review Article

\title{
Materials Selection as an Interdisciplinary Technical Activity: \\ Basic Methodology and Case Studies
}

\author{
M. Ferrante*, S.F. Santos, J.F.R. de Castro \\ Universidade Federal de São Carlos, Departamento de Engenharia de Materiais, \\ 13565-905 São Carlos - SP, Brasil
}

Received: January 24, 1999; Revised: April 7, 2000

\begin{abstract}
The technical activity known as Materials Selection is reviewed in its concepts and methodologies. Objectives and strategies are briefly presented and two important features are introduced and discussed; (i) Merit Indices: a combination of materials properties, which maximises the objectives chosen by the designer and (ii) Materials Properties Maps: a bi-dimensional space whose coordinates are pairs of properties in which materials can be plotted and compared directly in terms of their merit indices. A general strategy for the deduction of these indices is explained and a formal methodology to establish a ranking of candidate materials when multiple constraints intervene is presented. Finally, two case studies are discussed in depth, one related to materials substitution in the context of mechanical design and a less conventional case linking material selection to physical comfort in the home furniture industry.
\end{abstract}

Keywords: materials selection, design; merit indices

\section{Introduction}

Engineers are trained to execute many different technical activities such as product design, process optimisation, plant maintenance and quality control, to name only a few. Historically, their expertise has been adjusted to demand by specialisation, in other words, mechanical, materials, industrial, chemical, electronic engineers etc., each acting within defined boundaries were until recently the typical human resource of all industrial/technical activities. Presently, however, in the scenario designed by modern industry the various technical fields overlap to a large extent. An important consequence of such impingement is that interdisciplinary knowledge is replacing strict specialisation and that decision-making processes are becoming increasingly complex leading to the aggregation of experts into teamwork. This means that professionals with different bodies of knowledge combine around a common problem, each providing its own expertise and, above all, communicating across it. The quality of this communication is determinant for the success of the whole enterprise and the main ingredient of that quality is the sharing and cross-fertilisation of both knowledge and information.

One of the technical activities in which interdisciplinarity and the benefits of teamwork are particularly strong is materials selection (MS). Within this interdisciplinarity two levels may be distinguished. The former is essentially internal; in order to be carried out properly, MS must be combined with process selection (PS), thus integrating materials, metallurgical, process and production engineers. The external level is more complex, since multiple interactions between design, materials, process capabilities, market size and nature, cost, environmental impact and other constraints, must be taken into account ${ }^{1}$. Thus, at the internal level these interactions are easily identified: for instance, material and process; mechanical properties and stress analysis; product design and shaping characteristics of the candidate materials, etc. On the other hand, an example of interdisciplinarity at the external level would be the cost assessment of a particular product as a function of population buying power patterns forecast. It is apparent that the expertise required for decision-making processes of this nature must be found outside the strictly technical team, which was sufficient only at the internal level. Thus, depending of the economic weight of the decision it would not be exaggerated to say that engineers, marketing specialists, psychologists, demographist and, perhaps, political scientists are all equally necessary for such entreprise.

Figure 1 shows schematically the interactive or feedback relationships joining design and MS around a common objective. Interactions are limited to the level of the present paper's concern, that is, the internal level. In this figure, it

*e-mail: ferrante@power.ufscar.br 
can be seen that product development, or the path going from idea to final product, is composed by a number of steps each associated to a particular form of knowledge and in need of different information patterns. Starting with market requirements, both cost and production scale can be defined thus providing inputs for decisions such as process selection and expected cost composition (manufacture, material, maintenance etc.). Moreover, knowledge of the demands that the environment exerts upon the product is linked to overall perfomance, life expectancy, maintenance requirementes and Materials Selection. For instance, a corrosive environment can create conditions for stress-corrosion attack, which has to be taken into account by design and stress analysis calculations. Finally, it must be borne in mind that the above mentioned activities depend on information related to expected perfomance, which in its turn is related to market requirements. In conclusion, Fig. 1 summarises the steps needed to produce an object and show that four major considerentions are involved in such task: (i) the function what the object is supposed to do; (ii) in what environment he is expected to operate; (iii) for how long; and (iv) how much the solution will cost compared with the market expectation in this sense.

The evolution of MS from an empirical task to an organised, almost scientific methodology, took place mainly during the late eighties. Before that, texts on the subject usually consisted of tedious lists of materials properties and applications, with a few generalising attempts such as: materials for high temperature applications; materials for wear resistance, and so forth. One of the first modern textbooks on MS was written by Charles and Crane in 1978, and already contained criteria within which that activity should be conducted and rules regarding decisionmaking procedures ${ }^{2}$. But it was only in 1989 that a new philosophy of MS was proposed and soon adopted by most practitioners of the field ${ }^{3}$. This new approach is due to

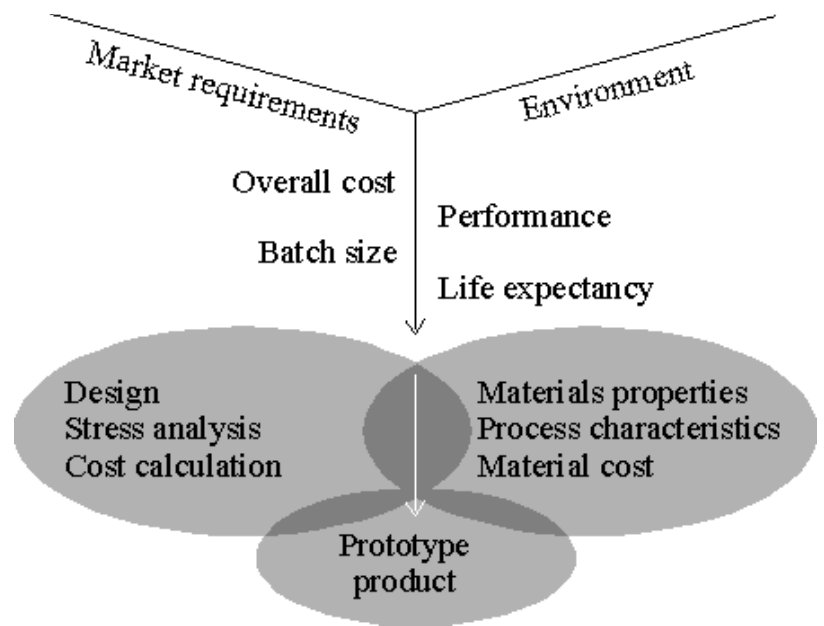

Figure 1. The interactions between design, product and materials selection.
Professor Michael F. Ashby and it is based on the concept of the "Materials Properties Maps" (MPM), through which a wide range of materials can be compared in terms of their properties and response under different physical situations. Finally, the integration of MPM with the concept of Merit Indices (MI) added sufficient generality to the Ashby approach, which can be considered as the most complete and useful to date. Although developed as an educational tool ${ }^{4}$ it can also be applied to industrial situations.

This paper attempts to review the MS basic methodology, illustrating it with two original case studies, one essentially technical in the engineering sense, the other chosen in such a way as to introduce the relationship between material and design in the creative sense.

\section{Main Concepts of Materials Selection}

\subsection{Objectives and strategies}

Materials Selection activities can be carried out having multiple objectives in sight, each characterised by a different approach requiring its particular strategy. The following list, by no means complete, gives some examples:

- Cost Reduction: options can include change of construction materials, viz. polymers replacing steel in the automotive industry.

- Meeting New Service Conditions: change of pressure and temperature in a chemical reactor - upgrading of existing materials.

- Weight Reduction: this is a universal objective for everything which is mobile.

- Materials vs. Process: there are cases in which materials substitution must be carried out in order to allow the adoption of a more suitable fabrication process.

- New Materials: complete redesign of the product or device may be necessary in order to fully exploit the new material properties.

- Materials and Aesthetic Design: apparently this is a subjective area, difficult even to qualify and as such better left to non-technical papers. However, it can be mentioned that a new product or the exploitation of new shape possibilities sometimes depends on choosing the right material and the most adequate shaping process. A good illustration of the relationship between materials and aesthetics is given by the domestic appliances, whose design evolution during the last fifty years has been quite remarkable.

\subsection{Macroscopic and microscopic approaches}

From the above considerations it can be concluded that MS is the point of convergence of various objectives and different technical competence. Also, the association of material with process introduces further complexity since another level of constraint is thus added. These points can be illustrated by observing the complete cycle of a MS event, in which, for instance, a particular aluminium alloy 
was selected. Besides mechanical properties and geometry, a number of process-dependent specifications must be taken into account. The complexity of that MS event can be appreciated remembering, that there are approximately 600 different aluminium alloys and a variety of manufacturing and fabrication routes (casting, extrusion, forging, stamping, welding, riveting). If we take into account that within the mechanical properties range of the chosen alloy a number of steels, titanium alloys, composites and magnesium alloys can also fit, it can be concluded that the initial choice is enormously large. Figure 2 is a schematic of the sequence of steps and constraints, which are common to the majority of MS events. From the above it follows that a macroscopic approach has to be adopted on the left-hand side of the figure so as not to miss any opportunity in terms of candidate materials. This has to be gradually changed towards a more detailed procedure - the microscopic approach - while the decision-making process moves towards the right hand side.

\subsection{Materials properties maps - merit indices}

In order to fully exploit the macroscopic approach it is necessary to consider a large number of candidates. This can be provided by materials properties tables but, in this case, the opportunity of simultaneous analysis is usually lost. Thus, graphic data bases are more suitable. Also, the example given in the previous section shows that multiple criteria or requirements have to be negotiated. This introduces the need to combine properties in such a way as to ideally at least - meet simultaneously the constraints. Since graphs are usually bi-dimensional, it results that only two constraints can be tackled at a time. For instance, taking two very common requirements in mechanical design - high stiffness and low weight - it is intuitive that the ratio Young Modulus (E) / density ( $\rho$ ) will take care of both requirements. This ratio is a simple example of MI. Taking the same properties as XY coordinates, materials can be plotted in the resulting space thus forming a MPM, see Fig. 3. The three lines superimposed on it represent constant MI values with the form $\mathrm{E}^{\mathrm{n}} / \rho$, where $\mathrm{n}$ depends of the loading mode,

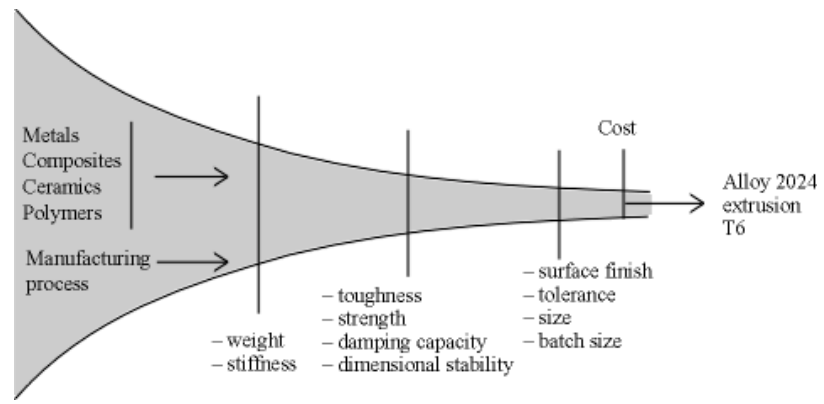

Figure 2. The funnel-like shape of a typical MS procedure and some decision steps along the way. Some constraints related to perfomance and to properties are shown. The decision-sequence along the the horizontal axis is only demonstrative; for instance, cost could have been the first gate. see Table 1. In order to use efficiently the map as a MS tool, two different "reading modes" should be adopted:

- Diagonal reading: used to locate materials, represented by $(\mathrm{x}, \mathrm{y})$ points in the XY space, with respect to the constant MI line of interest. Thus, materials lying along a particular line will have the same MI; those above and below will have higher and lower values, respectively.

- Orthogonal reading: Stiffness is proportional to E; therefore the $\mathrm{Y}$ axis can inform whether for the same performance (equal deflection under a given force for the same span) material A shall have either a smaller or a larger cross section (in other words, moment of inertia) than material B, C, D, etc. For instance, a minimum E, which translates into the maximum allowed cross-section, can be taken as a very simple form of MI.

Table 1 shows that MI are specific to articular loading modes. For its deduction a strategy will be shown in which objectives are correlated to restrictions. Therefore, taking a project in which the stiffness of a mechanical component is

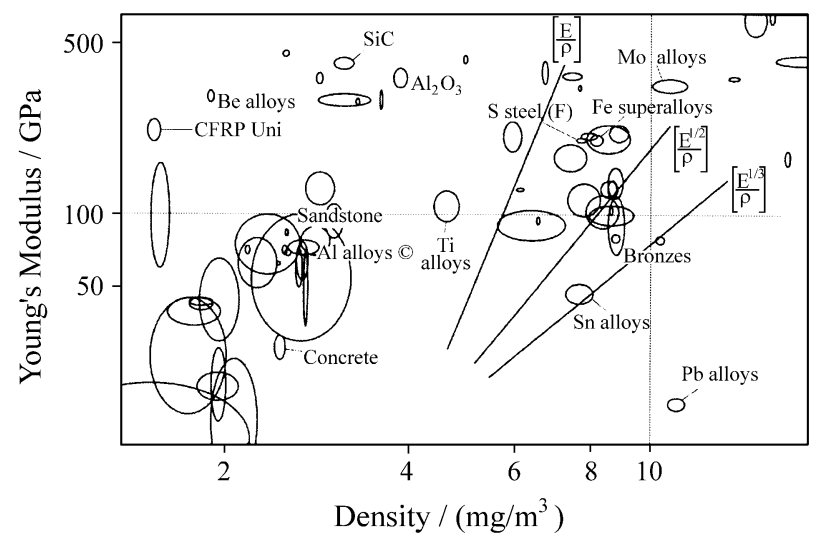

Figure 3. Material Property Map for Young modulus and density ${ }^{5}$. For clarity only some materials were labelled. It can be seen that for the same performance a steel tie working in tension $(\mathrm{E} / \rho)$ will be heavier than one made of titanium. However, orthogonal reading reveals that the latter will have a much larger cross section than the former.

Table 1. Merit indices for different loading modes when stiffness is the main project criteria.

\begin{tabular}{llc}
\hline Loading Mode & \multicolumn{1}{c}{ Shape } & Merit Index \\
\hline \multirow{2}{*}{ Bending } & Rod & Tube \\
& Plate & $\mathrm{E}^{1 / 3} / \rho$ \\
\hline Tension & Tie & $\mathrm{E} / \rho$ \\
\hline \multirow{2}{*}{ Buckling } & Column & $\mathrm{E}^{1 / 2} / \rho$ \\
& Plate & $\mathrm{E}^{1 / 3} / \rho$ \\
\hline Torsion & Rod, Tube & $\mathrm{G} / \rho$ \\
\hline \multirow{2}{*}{ Internal pressure } & Cylindrical pressure vessel & $\mathrm{E} / \rho$ \\
& Spherical pressure vessel & $\mathrm{E} /(1-\mathrm{v}) \rho$ \\
\hline
\end{tabular}


the design criteria, the MI strategy can be summarised as follows:

- Objective: minimum weight;

- Restriction: stiffness (maximum or specified deflection).

Initially, it is necessary to write an equation for the objective and another for the restriction or constraint. The deduction of the above chosen MI will be demonstrated below using the simple example of a beam subjected to a bending load and to a specified deflection. Dimensions (except the free dimension, which is obtained by design calculation) and loads are known.

Beam - circular section, radius $r$ and length $L$. The objective and the restriction are given respectively by the two equations:

$$
\begin{aligned}
& \mathrm{m}=\pi \mathrm{r}^{2} \mathrm{~L} \rho \\
& \delta=\frac{\mathrm{FL}^{3}}{\mathrm{CE} \mathrm{I}}
\end{aligned}
$$

where $\mathrm{m}$ is the mass, $\mathrm{F}$ the load, $\mathrm{C}$ a constant which depends of the loading conditions and I the polar moment of inertia, equal to $\pi r^{4} / 4$ for circular sections. Substituting I in Eq. 2, we have:

$$
\delta=\frac{F L^{3}}{C_{1} E \pi r^{4}}
$$

where $\mathrm{C}_{1}$ is equal to $\mathrm{C} / 4$.

Next comes the identification of the free variable, defined as the dimension whose change affects the performance of the product or component. In the present case $\mathrm{L}$ is determined by the project, thus the free variable is $r$, and eliminating it between Eq. 1 and 3 results:

$$
\mathrm{m}=\left(\frac{\mathrm{F}}{\delta}\right)^{1 / 2}\left(\frac{\pi \mathrm{L}^{5}}{\mathrm{C}_{1}}\right)^{1 / 2}\left(\frac{\rho}{\mathrm{E}^{1 / 2}}\right)
$$

It must be observed that Eq. 4 has been rearranged so as to isolate terms identified with performance, dimensions and materials properties, respectively. Since minimisation of mass is the objective, the relevant MI is obtained inverting the last term within square brackets and materials can now be compared in terms of $\mathrm{E}^{1 / 2} / \rho$. Thus, referring to Fig. 3 , diagonal reading means to locate the candidate materials with respect to a family of lines with slope 2 . Choices can then be carried out with the minimum weight objective in mind. It must be remembered that different MI values mean that:

- The materials have different weight $\left(\mathrm{m}_{\mathrm{i}}\right)$ for the same performance

- Conversely, if the components have the same weight their performance will be different

- There is a relationship between these parameters such as that:

$$
\frac{\mathrm{m}_{1}}{\mathrm{~m}_{2}}=\frac{(\mathrm{MI})_{2}}{(\mathrm{MI})_{1}}=\frac{(\text { performance })_{2}}{(\text { performance })_{1}}
$$

However, choices made comparing ratios such as $E^{\mathrm{n}} / \rho$ do not allow any insight into abolute values of properties, therefore the orthogonal reading mode is necessary to make sure that, although achieved the minimum mass objective, sections will not be larger than the absolutely necessary.

\subsection{Case studies}

Two examples will be presented here. The former deals with the rotor of a laboratory centrifuge, within the context of product development. The problems which this example entails are typically structural/mechanical and no emphasis has been placed on cost issues, since very specialised and complex cost-assessing techniques are available to the professional $^{6,7}$. The second example is a typical case in which "the material is the product". It is a design piece of furniture in which comfort and aesthetics can be combined by a correct choice of the material. Its main purpose is to show how freedom of design and functionality can be combined and enhanced by that choice.

Centrifuge: this is a machine rotating at very high speeds for solid-liquid or liquid-liquid separation. It normally consists of a motor driving a rotor which carries a number of glass or plastic tubes. Two different rotor designs are available: the fixed angle rotor, shaped as a wheel with peripheral orifices in which the tubes are fitted, and the swing out rotor shown in Fig. 4. While in the former design the tubes are kept at a fixed angle, generally between 35 and 45 degrees, in the latter the centrifugal force maintains the tubes horizontal. Shape and typical dimensions of a swing rotor are shown in Fig. 4.

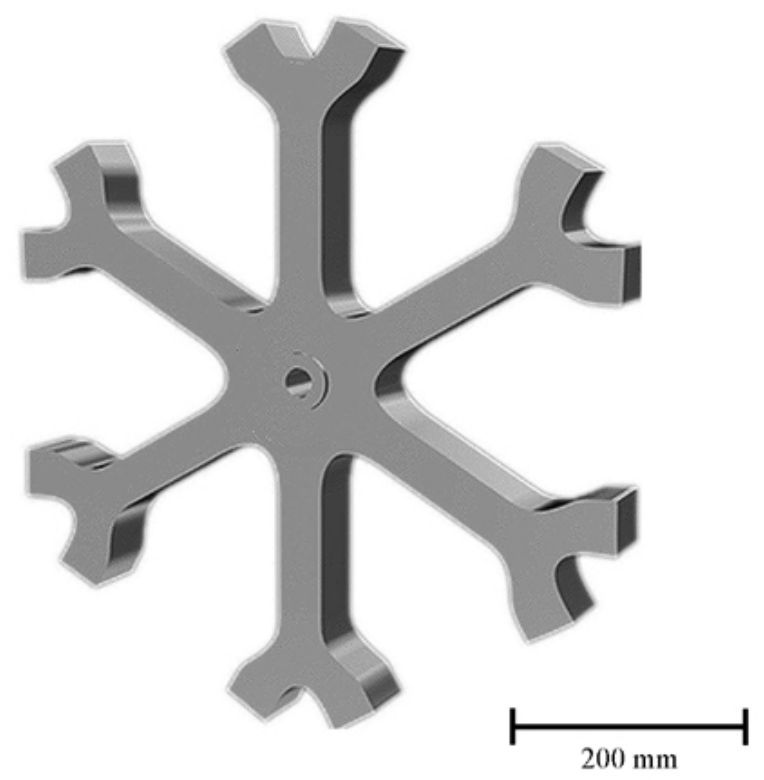

Figure 4. Schematic of a swing rotor. 
The MS procedure is as follows

Objective: maximum performance, that is maximum rotational speed with minimum weight of the rotor.

Restriction: must resist to centrifugal forces without rotor failure.

Calculation of the MI - beginning with the definition of centrifugal force $\left(\mathrm{F}_{\mathrm{c}}\right)$, we have:

$$
\mathrm{F}_{\mathrm{c}}=\frac{\mathrm{m} \mathrm{V}^{2}}{\mathrm{r}}
$$

where $\mathrm{m}$ and $\mathrm{r}$ are the mass and length of the arm, respectively, and $\mathrm{V}$ is its tangential speed.

For a small mass element $(\mathrm{dm})$ at a distance $r$ from the centre of the rotor, we have:

$$
\begin{aligned}
& \mathrm{dF}_{\mathrm{c}}=\frac{\mathrm{dm} \mathrm{V}^{2}}{\mathrm{r}} \\
& \mathrm{dm}=\rho \mathrm{Adr}
\end{aligned}
$$

and

$$
V^{2}=(2 \pi r)^{2} n^{2}
$$

where $\mathrm{A}$ is the cross section of the arm. Therefore Eq. 6 can be written

$$
\mathrm{dF}_{\mathrm{c}}=\frac{\rho\left(\mathrm{mg} / \mathrm{m}^{3}\right) \mathrm{dr}(\mathrm{m})(2 \pi \mathrm{r})^{2}\left(\mathrm{~m}^{2}\right) \mathrm{n}^{2}\left(\mathrm{~s}^{-2}\right)}{\mathrm{r}(\mathrm{m})}
$$

The total centrifugal force acting on the arm is given by:

$$
\mathrm{F}_{\mathrm{Tc}}=4 \pi^{2} \rho \mathrm{A} \mathrm{n}^{2} \int_{0}^{\mathrm{r}} \mathrm{r} \mathrm{dr}=2 \pi^{2} \mathrm{~A} \mathrm{n}^{2} \mathrm{r}^{2}
$$

and inserting the value of $\mathrm{V}^{2}$, the final expression for the total centrifugal force can be written as:

$$
\mathrm{F}_{\mathrm{Tc}}=\frac{\rho \mathrm{A}}{2} \mathrm{~V}^{2}
$$

The restriction here adopted is analytically expressed by $\mathrm{F}_{\mathrm{Tc}} \leq \sigma \mathrm{A}$. Inserting this relation into Eq. 8 and rearranging terms we have:

$$
\frac{\sigma}{\rho} \geq \frac{\mathrm{V}^{2}}{2}
$$

Thus, the MI sought for is given by the ratio.

$$
\left[\frac{\sigma}{\rho}\right]
$$

and a line of slope equal to 1 on the $\sigma$ vs. $\rho$ graph results. For the present case the relevant operational characteristics are:

- rotational speed (n): $6000 \mathrm{rpm}$.

- length of the rotor arms (r): $20 \times 10^{-2} \mathrm{~m}$. and we have:

$$
\mathrm{V}=2 \times[6000 / 60(\mathrm{sec})] \pi \times 10 \times 10^{-2}(\mathrm{~m})=126 \mathrm{~m} \mathrm{~s}^{-1}
$$

It is reasonable to assume a safety factor equal to 4 , with respect to $\mathrm{F}_{\mathrm{Tc}}$. Therefore, Eq. 9 is multiplied by 4, and in this particular case we have:

$$
\mathrm{MI} \geq 2 \mathrm{~V}^{2} \geq 2 \times 126^{2} \cong 32 \times 10^{3}\left(\mathrm{~m} \mathrm{~s}^{-1}\right)^{2}
$$

Thus, in order to combine the objective with the restriction, the above requirement must be obeyed.

Figure 5 is a MPM for the pair of materials properties $\sigma$ and $\rho$. Superimposed on it there is a line with slope equal to 1 , the IM line, along which a constant value of $32 \times 10^{3}$ is maintained. All materials above it are suitable candidates in terms of the objective and restriction initially adopted. Therefore, from Fig. 5 it can be seen that for the stress conditions here calculated most structural materials are suitable. This conclusion, borne from the diagonal reading mode, is not much selective, but in this particular case, since the objective is maximum performance (maximum rotational speed), the larger the distance from the IM line (in other words, the larger the MI), the higher the performance for a given weight. A number of restrictive requirements can now be made in order to narrow the selection procedure, see Table 2.

Justifications: (i) Toughness: the figure of $20 \mathrm{MPa} \mathrm{m}^{1 / 2}$ is commonly quoted as a lower bound for structural design; (ii) Process: the product in analysis,

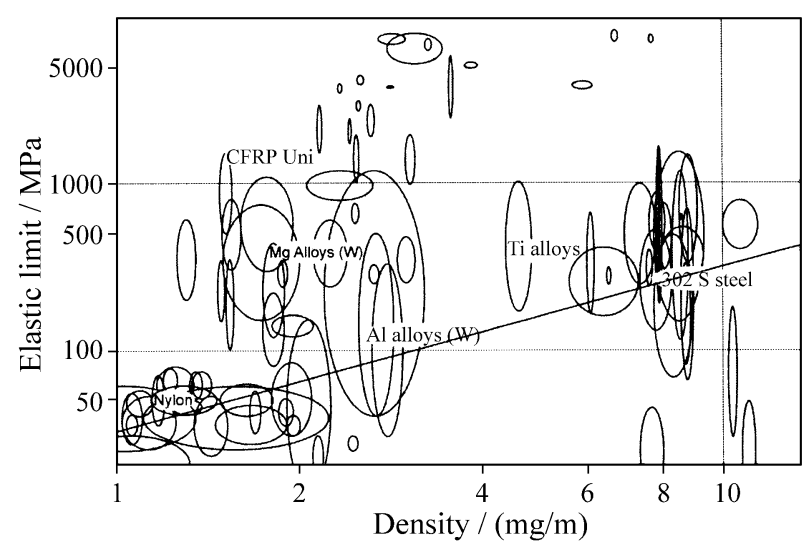

Figure 5. Material property map ( $\sigma v s . \rho)$ for the centrifuge rotor material selection.

Table 2. Initial requirements.

\begin{tabular}{lc}
\hline Item & Requirement \\
\hline Toughness & $>20 \mathrm{MPa} \mathrm{m}^{1 / 2}$ \\
Process & avoid casting \\
Availability/price stability & medium to high \\
Normalisation & desirable \\
Price & low \\
\hline
\end{tabular}


Table 3. Candidate materials, respective properties and MI.

\begin{tabular}{lccccc}
\hline Material & $\sigma_{\mathrm{y}}[\mathrm{MPa}]$ & $\rho\left[\mathrm{Mg} / \mathrm{m}^{3}\right]$ & $\mathrm{K}_{\mathrm{Ic}}\left[\mathrm{MPam}^{1 / 2}\right]$ & $\sigma_{\mathrm{f}}[\mathrm{MPa}]$ & {$[\sigma / \rho]\left[\left(\mathrm{m} \mathrm{s}^{-1}\right)^{2} \mathrm{x} \mathrm{10^{3 } ]}\right.$} \\
\hline Aluminium 2024 T4 & 500 & 2.8 & 35 & 155 & 178 \\
Ti-6Al-4V & 850 & 4.4 & 100 & 550 & 193 \\
Carbon fibre composite* & 1000 & 1.5 & 33 & 700 & 670 \\
302 stainless steel & 600 & 7.8 & 90 & 350 & 77 \\
Magnesium ZC 71** & 320 & 1.9 & 17 & 180 & 168 \\
Nylon 6/6 & 60 & 1.1 & 2 & 25 & 55 \\
\hline
\end{tabular}

(*) Carbon Fibre XP EPC F001; 55\% C; (**) Mg-6.5Zn-1.25Cu-0.75 Mn.

requires a doubling of the safety factor when casting processes are employed.

Therefore, the materials listed in Table 3, together with their respective properties and the MI above developed can be selected from Fig. 5. Fatigue strength $\left(\sigma_{\mathrm{f}}\right)$ was also included, although no minimum value has been established.

From the above data the Mg alloy can be excluded due to its low fracture toughness. As for the remaining candidates the following comments can be made:

Aluminium: exhibits only the third best performance, for a given weight (or vice-versa). It also has an acceptable endurance limit, and a sufficient toughness level. Normalisation and low cost are positive assets.

Ti-6Al-4V alloy: good strength, toughness and fatigue resistance, but moderate MI. Very well known alloy, but high cost.

Composite - carbon fibres: best merit indices for both strength and fatigue limit. Reasonable toughness but very high cost. Uncertainties regarding the properties may call for a reassessment of the safety factor.

AISI 302 stainless steel: low mechanical resistance and high density. Thus, following Eq. 5 a stainless steel rotor will be 8,7 times heavier than the same part made with the XP EPC F001 composite. Moderate price, reproducible properties.

Nylon 6/6: low merit index but still sufficient for the application. Very low fracture toughness, but with materials having low Young modulus, such as polymers, a different approach to fracture design must be employed. Thus, due to its high flexibility, what matters in the present case is the ratio [ $\left.\mathrm{K}_{\mathrm{Ic}} / \mathrm{E}\right]$, which is acceptable. If dimensional stability is a stringent requirement it should be borne in mind that water absorption $(\sim 1.0 \%)$ can be a problem. Very heavy due to its large section.

From the above comments it can be seen that a straightforward choice is still difficult, one material being superior to the others under a particular set of requirements but inferior when different aspects are considered. Additionally, cost is still not in question here although it constitutes one of the more important and complex decision factors. As for the properties, besides maximising performance per unit weight, as expressed by $[\sigma / \rho]$ or $\left[\sigma_{f} / \rho\right]$, the rotor must also dampen vibrations and ideally its resonance frequency $f_{\mathrm{r}}$ should not coincide with the rotational speed, viz., $100 \mathrm{~Hz}$.

From the data it can be concluded that vibrations are efficiently dampened by the composite, followed by the aluminium alloy. As for the resonant frequencies, all materials are acceptable since $f_{r}$ is always higher than $100 \mathrm{~Hz}$.

Sofa: modern furniture is an expanding industry which combines aesthetics with physical comfort. Because focus is mainly on design, cost and fabrication methods, material selection tends to be based more on past experience and the awareness of new materials is somewhat low. Figure 6 shows a modern design in which the chair is almost entirely constituted of foamed polymer. It can be assumed that physical comfort ${ }^{\#}$ depends of the correct value and proper balance of the following properties:

- softness - contributes to limit the pressure on the seated body

- stiffness - prevents excessive "sinking" of the body in the chair, mainly during the initial stages of sitting.

Translating these characteristic into mechanical properties it can be said that under the compressive stresses caused by the user, strain must be limited to a pre-determined

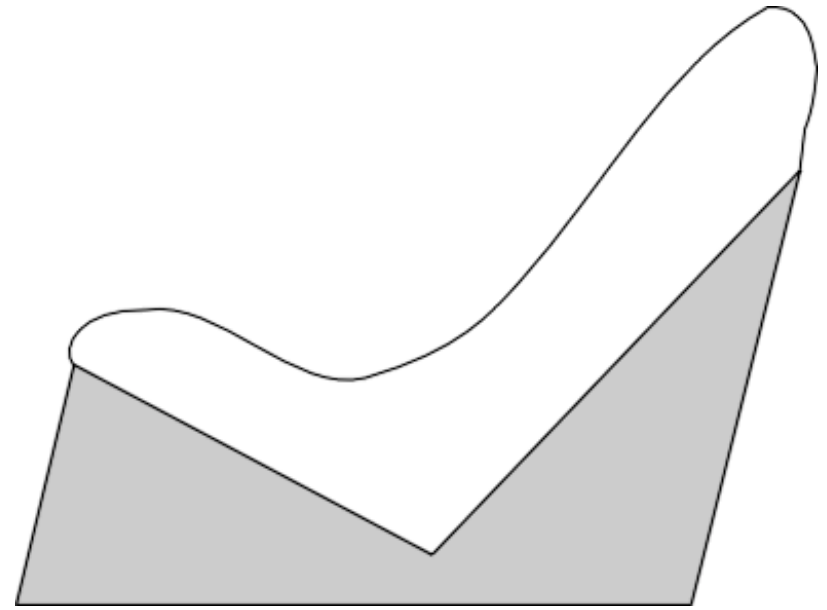

Figure 6. Soft chair of modern design made of expanded foam contained in a metallic frame. 
value. Therefore the selection will be based on bulk modulus (K). Other relevant factors are thermal conductivity and water absorption of the foam. It can be assumed that for physical comfort the former characteristic must be kept high, whilst low values of the latter are preferable.

From ergonomic data ${ }^{8}$ the pressure exerted by a body during the early stages of sitting can be estimated as between 0.4-0.5 MPa. Taking the latter value and assuming that for the seat showed in Fig. 6 a comfortable initial deformation would be close to $80 \%$, we have:

$$
\mathrm{K}=\frac{\sigma}{\varepsilon}
$$

And inserting numerical values:

$$
\frac{0.5 \mathrm{MPa}}{0.8}=0.63 \mathrm{MPa}
$$

therefore, we have:

$$
\frac{0.5 \mathrm{MPa}}{0.63 \times 10^{-3} \mathrm{GPa}}=794
$$

and the problem reduces to the selection of foams having bulk modulus equal to $\sim 0.60 \mathrm{MPa}$, in other words the ratio compressive stress/bulk modulus [MPa/GPa] must be close to 800 . This behaviour can be combined with another selection criteria, thermal conductivity for instance, and Fig. 7 is a material properties map in the space $\sigma / \mathrm{K}$ (combined properties) against thermal conductivity. It can be seen that in terms of deformation behaviour three materials are acceptable: Polyurethane elastomer foam, foamed polymer (medium density) and foamed polymers (high density). However, this last material is more suitable under the thermal behaviour point of view, since a cooler environment for the user is provided but is bordeline with respect to $[\sigma / \mathrm{K}]$.

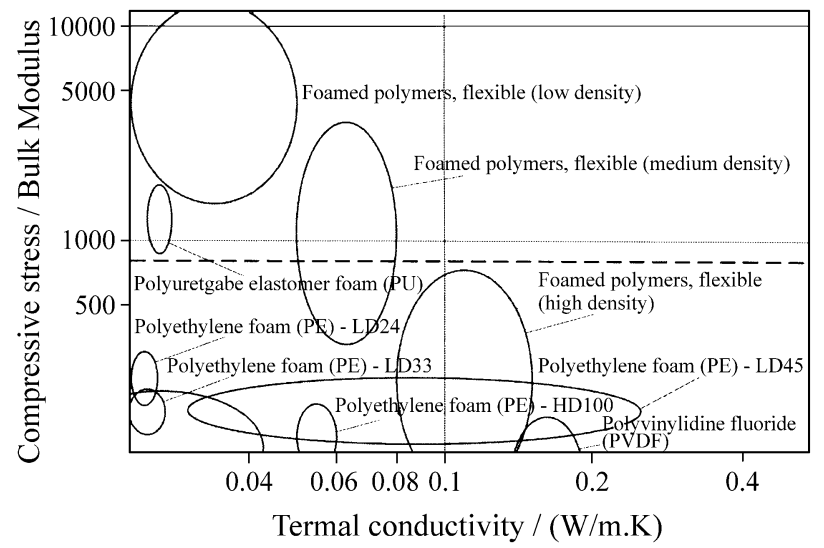

Figure 7. Materials Property Map from which selection of foams combining a given deformation under load with high thermal conductivity, can be carried out.
Additionally, it can be shown that the high density foamed polymer exhibits a reasonably low level of water absorption $(\sim 0.1 \%)$ and its price is comparable to those of the other candidate materials ${ }^{5}$.

\subsection{Formalisation of the material selection procedure}

The two examples here examined show that any MS procedure begins with a list of the materials relevant properties or characteristics. The next step will be the organisation of these characteristics according to their individual importance, in other words each property or MI will be associated to a "weighting factor". The establishment of such factors is a typical multidisciplinar task, involving material and production engineers, plus design, marketing and cost analysis specialists. It is a very delicate step in which the boundary conditions of the whole project have to be established and quantified according to the available data. At this stage the basic design features of the product will be kept constant but a large degree of freedom must still be adopted with regard to materials, process, design solutions, assembly and joining techniques and so on. This stage of the project will be called formalisation of the material selection procedure (FMSP) and an example based on the centrifuge rotor case study will be presented.

Generally FMSP is carried out by making use of the so called Decision Matrices (DM) which can be defined as organised procedures by which materials are ranked prior to a selection decision. Different DM forms have been developed, namely the Pugh method ${ }^{9}$, the Dominic method $^{10}$ and the Pahl \& Beitz method ${ }^{11}$, all having in common the comparison of alternatives according to a number of criteria whose priority is established by the weighting factors. These factors are determined by technical reasons, by customer preferences, or both.

Of the above mentioned DM forms, only the Pahl \& Beitz is quantitative, and the relevant requirements are expressed by values scales as shown below:

\begin{tabular}{lccccc}
\hline $\begin{array}{l}\text { Value } \\
\left(v_{\text {ij }}\right)\end{array}$ & 0 & 1 & 2 & 3 & 4 \\
Meaning & $\begin{array}{c}\text { Unsatis- } \\
\text { factory }\end{array}$ & $\begin{array}{c}\text { Just } \\
\text { tolerable }\end{array}$ & Adequate & Good & $\begin{array}{c}\text { Very } \\
\text { good }\end{array}$ \\
\hline
\end{tabular}

There is also a 11 points value scale, rating from absolutely useless to ideal. The Pahl \& Beitz method can be described as follows:

(i) The functional description of the product and the criteria or requirements are arranged on a "tree", composed by two or more levels. There are $\mathrm{n}$ criteria and $\mathrm{k}$ candidate materials;

(ii) The tree is similar to a conventional organogramme in which each level is subordinate to the higher order level. Therefore, criteria of the same order are arranged on horizontal lines, all belonging to the same level; 
(iii) Higher levels express the basic requirements. Lower levels contain secondary or ancillary selection criteria;

(iv) To each criteria it is assigned a value $\left(v_{\mathrm{ij}}\right)$, taken from the value scale given above. Therefore, there are (k) (n) values to assign;

(v) Each criteria is multiplied by a weighting factor $\left(\mathrm{w}_{\mathrm{ij}}\right)$ which is deployed besides it. In each level the sum of these factors equals 1 .

(vi) For any criteria of order $n$, with $n \geq 2$, the correspondent weighting factor $\left(\mathrm{w}_{\mathrm{ij}}\right)$ is the product of the weighting factor associated to the parent criteria of level immediately superior, that is, level (n-1), with the weighting factor associated to the criteria in consideration.

The final rating is given by summing all weighted values for the candidate material $i$ and is called the Overall Weighted Value $\left(\mathrm{OWV}_{\mathrm{i}}\right)$. It is customary to normalise $O W V_{i}$ 's to 1 by dividing by the maximum value $\left(v_{\max }\right)$ and the sum of the weighting factors. In this case we obtain the so called Weighted Property Index $\left(\mathrm{WR}_{\mathrm{i}}\right)$ whose formula is:

$$
\mathrm{WR}_{i}=\frac{\sum_{\mathrm{j}=1}^{\mathrm{n}} w \mathrm{j} \cdot v_{\mathrm{ij}}}{v_{\max } \sum_{\mathrm{j}}^{\mathrm{n}} \mathrm{w}_{\mathrm{j}}}
$$

where normally:

$$
\sum_{j=1}^{n} w_{j}=1
$$

The Pail \& Beitz method will be applied to the centrifuge rotor case study just examined. Thus, the functional description and relevant criteria can be arranged in three levels and the resulting tree is shown in Fig. 8.

Finally, Table 4 summarizes quantitatively the procedure already described from (i) to (vi). However, item (iv) can be advantageously modified by replacing the value scale with the correspondent MI. This removes the subjectivity associated to the assessment of qualities, such as "excellent", "adequate", etc. Thus, Table 4 employs only numerical values, which are closer to the relevant mechanical properties.

\section{Observations:}

(i) Toughness has been analysed in terms of critical defect size, which is proportional to $\mathrm{K}_{\mathrm{Ic}} / \sigma$ (where $\sigma$ is the elastic limit for ductile material and fracture strength for non-ductile ones);

(ii) Material cost is initially given in US dollars per kg. For a better estimate this cost was divided by $[\sigma / \rho]$, thus taking into account the weight difference between the candidate materials (see Eq. 5);

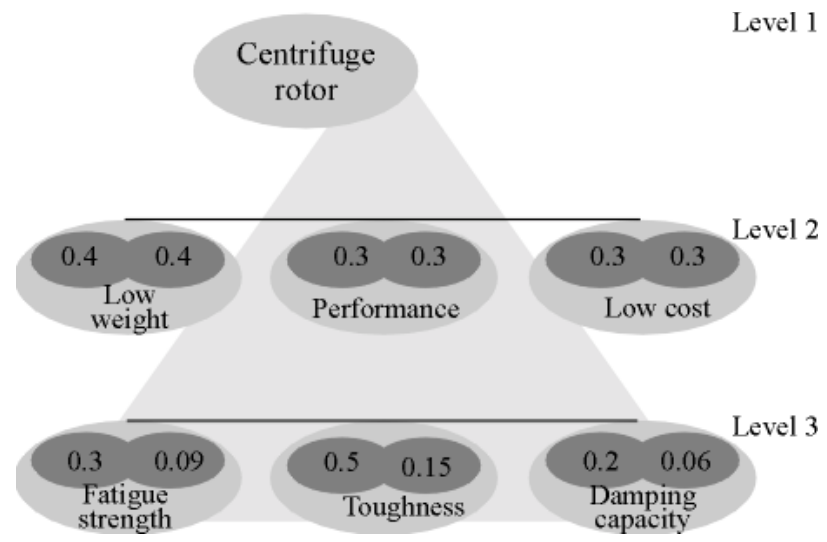

Figure 8. Pahl and Beitz decision matrix illustrating the functional description of the centrifuge rotor, the various criteria and the correspondent wheighting factors $\left(\mathrm{w}_{\mathrm{ij}}\right)$.

(iii) Normalisation of the data has been performed by dividing each value by the maximum figure.

As it can be seen the largest $\mathrm{WR}_{\mathrm{i}}$ belongs to the carbon fibres composite followed very closely by the AA2024 alloy. Other criteria can be added in order to get a more clear-cut decision. For instance, both raw material supply conditions and properties reliability are favourable to the Al alloy.

\section{Final Comments}

Materials selection is a technical activity that bridges a number of engineering areas, viz., design, cost, materials and processes, but can also be related to non-technical issues, such as marketing and techno-economic forecast. The systematic approach here presented is due to M.F. Ashby and today represents the most advanced methodology available. Originally conceived as an educational tool, its evolution into a very friendly-user software combined with a large number of technical data means that it can be applied to any real situation. Following the Ashby approach, this paper presented two case studies, which can be taken as the extremes of a broad spectrum going from a typically structural component to a domestic furniture item. In connection with the centrifuge rotor case study, the formalisation method here employed, see Table 4, can be considered as an improvement over the original Pahl and Peitz method, since only numerical data has been used instead of subjective value scales.

The use of the Materials Properties Maps embodies the macroscopic approach, and integrates the MI into the selection process. This has both didactic and practical advantages, and in the latter case it assures that no opportunity was lost by narrowing the selection process from the initial stages. Of course, there are more clear-cut cases in which choices will be restricted from the beginning to a particular family or sub-family of materials, such as aluminium alloys, heat resisting steels etc. Another practical constraint, which militates against the macroscopic approach, is the 
Table 4. (next page).

fact that industries invest large sums of money in some specific product, related machinery and technologies, thus limiting their choices.

The present paper did not discuss how process comes into the selection procedure, although it has been stressed that material and process are complementary. This omission is justified by the complexity of such integration which will need the linkage of data bases covering materials properties and process characteristics. For instance, it must be considered that real objects have properties which depend on both the material and the processing route, a feature which introduces further complexity to the SM task. Also, if one needs to make a very large number of small components, selecting the processing route may be more critical than the choice of material. Therefore, the present authors' opinion is that the development of a system that could select material and process combinations needs to be addressed by the professionals involved in MS systems.

\section{References}

1. Clark, J.P.; Roth, R.; Field III, F.R. Techno-Economic Issues in Material Selection, ASM Handbook, v. 20, Materials Selection and Design, p. 255, ASM International, 1997.

2. Charles, J.A.; Crane, F.A.A. Selection and Use of Engineering Materials, $2^{\text {nd }}$ ed., Butterworths, 1989.

3. Ashby, M.F. On the Engineering Properties of Materials, Acta Metall., v. 37 n. 5, 1989.

4. Ashby, M.F. personal communication.

5. Cambridge Materials Selector version 2.02, Granta Design LTD., 1994.

6. Hoult, D.P.; Meador, C.L. Predicting Product Manufacturing Costs from Design Attributes: a Complexity Theory Approach, n. 960003 Society of Automotive Engineerings, 1996.

7. Ostwald, P.F. American Machinist Cost Estimator, Penton Educational Division, Penton Publishing, 1988.

8. Osborne, D.J. Ergonomic at Work, John Wiley and Sons, 1982.

9. Pugh, S.; Total Design: Integrated Methods for Successful Product Development, Addison-Wesley, 1991.

10. Howe, A.E.; Cohen, P.R.; Dixon, J.R.; Simmons, M.K. Dominic: a Domain-Independent Program for Mechanical Design, The International Journal of Artificial Intelligence, v. 1, n. 1, 1986.

11. Pahl, G.; Beitz, W. Engineering Design; a Systematic Approach, Springer-Verlag, 1993. 
Table 4. Pahl and Beitz-based decision matrix for the centrifuge rotor.

\begin{tabular}{|c|c|c|c|c|c|c|c|c|c|c|c|}
\hline \multicolumn{4}{|c|}{ Criteria } & \multicolumn{2}{|c|}{$\begin{array}{c}\text { Material } 1 \\
\text { AA } 2024 \text { T6 }\end{array}$} & \multicolumn{2}{|c|}{$\begin{array}{l}\text { Material } 2 \\
\text { Ti-6Al-4V }\end{array}$} & \multicolumn{2}{|c|}{$\begin{array}{c}\text { Material } 3 \\
\text { C fibres composite }\end{array}$} & \multicolumn{2}{|c|}{$\begin{array}{c}\text { Material } 4 \\
\text { Nylon } 6 \\
\end{array}$} \\
\hline Primary consideration & No. & Evaluation criteria & $\begin{array}{l}\text { Weighting } \\
\text { factor }\left(w_{\mathrm{ij}}\right)\end{array}$ & $\begin{array}{l}\text { Value } \\
(\mathrm{IM})\end{array}$ & $\begin{array}{l}\text { Weighted } \\
\text { value }\end{array}$ & $\begin{array}{l}\text { Value } \\
\text { (IM) }\end{array}$ & $\begin{array}{l}\text { Weighted } \\
\text { value }\end{array}$ & $\begin{array}{l}\text { Value } \\
(\mathrm{IM})\end{array}$ & $\begin{array}{l}\text { Weighted } \\
\text { value }\end{array}$ & $\begin{array}{l}\text { Value } \\
(\mathrm{IM})\end{array}$ & $\begin{array}{l}\text { Weighted } \\
\text { value }\end{array}$ \\
\hline Weight (0.4) & 1 & Low weight & 0.4 & 178 & 71.2 & 193 & 77.2 & 670 & 268 & 55 & 22 \\
\hline \multirow[t]{3}{*}{ Perfomance $(0.3)$} & 2 & Fatigue strength & 0.09 & 55 & 5.0 & 125 & 11.2 & 470 & 42.3 & 23 & 2.1 \\
\hline & 3 & Toughness & 0.15 & 0.07 & $10.5 \times 10^{-3}$ & 0.12 & $17.6 \times 10^{-3}$ & 0.03 & $4.5 \times 10^{-3}$ & 0.03 & $4.5 \times 10^{-3}$ \\
\hline & 4 & Damping $\left(\eta \times 10^{-3}\right)$ & 0.06 & 2.5 & 0.15 & 1.0 & 0.06 & 1.5 & 0.09 & 0.02 & 0.0012 \\
\hline \multirow[t]{8}{*}{ Cost (0.3) } & 5 & US\$ / proportional weight & 0.3 & 0.016 & $4.8 \times 10^{-3}$ & 0.210 & $63 \times 10^{-3}$ & 0.150 & $45 \times 10^{-3}$ & 0.082 & $25 \times 10^{-3}$ \\
\hline & & Criteria/ consideration & \multicolumn{9}{|c|}{ Proportional values } \\
\hline & & 1. Low weight & \multicolumn{2}{|r|}{27} & \multicolumn{2}{|r|}{29} & \multicolumn{2}{|r|}{100} & \multicolumn{3}{|c|}{8} \\
\hline & & 2. Fatigue strength & \multicolumn{2}{|r|}{12} & \multicolumn{2}{|r|}{26} & \multicolumn{2}{|r|}{100} & \multicolumn{3}{|c|}{5} \\
\hline & & 3. Toughness & \multicolumn{2}{|r|}{60} & \multicolumn{2}{|r|}{100} & & 26 & \multicolumn{3}{|c|}{26} \\
\hline & & 4. Damping capacity & \multicolumn{2}{|c|}{100} & \multicolumn{2}{|r|}{40} & & 60 & \multicolumn{3}{|c|}{1} \\
\hline & & 5. Proportional cost & \multicolumn{2}{|r|}{8} & \multicolumn{2}{|r|}{100} & & 71 & \multicolumn{3}{|c|}{40} \\
\hline & & $\mathrm{WR}_{\mathrm{i}}=[(1+2+3+4+(100-5)] / 5$ & \multicolumn{2}{|r|}{58} & \multicolumn{2}{|r|}{39} & & 63 & \multicolumn{3}{|c|}{20} \\
\hline
\end{tabular}

